\title{
Histoplasmoma pulmonar no Rio Grande do Sul
}

\author{
Pulmonary histoplasmoma in Rio Grande do Sul
}

\author{
Gisela Unis ${ }^{1}$, Karla Lais Pêgas ${ }^{1}$ e Luiz Carlos Severo ${ }^{1}$
}

\begin{abstract}
RESUM0
Foram revisados dezesseis prontuários de pacientes que apresentavam nódulo pulmonar à radiografia de tórax diagnosticados como histoplasmoma através de achado histopatológico demonstrando elementos fúngicos compatíveis com Histoplasma capsulatum var capsulatum no Rio Grande do Sul. Revisada a literatura brasileira. Todos os pacientes eram brancos, nove eram homens, a média de idade foi de 50,8 anos. Seis $(37,5 \%)$ pacientes tinham história epidemiológica sugestiva de contato com fungo. Os nódulos variaram de 0,7 a 2,7 cm de diâmetro, apresentavam-se como granuloma com necrose caseosa. Doze (75\%) pacientes apresentavam nódulo solitário. 0 histoplasmoma como regra se apresenta como nódulo solitário com até três centímetros de diâmetro em paciente assintomático. 0 diagnóstico é realizado por exame histopatológico com coloração especial para visualização do fungo, método de Gomori-Grocott com metenamina argêntica. Cultivo ou testes soromicológicos não contribuem para o diagnóstico.
\end{abstract}

Palavras-chaves: Histoplasmoma. Nódulos pulmonares. Histoplasma capsulatum var. capsulatum. Rio Grande do Sul. Brasil.

\begin{abstract}
A retrospective analysis of sixteen patients with pulmonary nodules diagnosed as histoplasmoma by histopathologic evidence of Histoplasma capsulatum var capsulatum was done in an endemic area for histoplasmosis. Brazilian literature was reviewed. All patients were white, nine were men, mean age at diagnosis was 50.8 years. Six (37.5\%) patients had a relevant epidemiologic story. The pulmonary nodules were $0.7-2.7 \mathrm{~cm}$ in diameter, presenting as granulomatous lesions with caseous necrosis. Twelve (75\%) patients had solitary nodules. Histoplasmoma as a rule is a solitary nodule of up to $3 \mathrm{~cm}$ in an asymptomatic patient. The diagnosis is made from histopathologic evidence of the fungus, which is accomplished using a special fungus stain, Gomori-Grocott methenamine silver. Cultures or seromycology do not help the diagnosis.
\end{abstract}

Key-words: Histoplasmoma. Pulmonary nodules. Histoplasma capsulatum var. capsulatum. Rio Grande do Sul. Brazil.

Nódulos pulmonares têm como diagnóstico diferencial neoplasia e doenças granulomatosas. Quando de origem granulomatosa, infecções micobacterianas, fúngicas e uma variedade de lesões não infecciosas devem ser distinguidas uma vez que apresentam tratamentos específicos ${ }^{18}$. 0 diagnóstico de vasculites primárias do pulmão como granulomatose de Wegener e sarcoidose é dificultado devido à falta de envolvimento extrapulmonar característico das vasculites sistêmicas. A utilização de tratamento empírico, embora freqüente, é inapropriada, já que além de ineficiente, muitas vezes é deletéria. 0 histoplasmoma é causa freqüente de nódulos pulmonares no Brasil ${ }^{111}$. A proposta de uma rotina de investigação de nódulos pulmonares justifica este trabalho.

\footnotetext{
1. Programa de Pós-Graduação em Medicina: Pneumologia, Universidade Federal do Rio Grande do Sul, Porto Alegre, RS.

Trabalho realizado como parte de Doutorado realizado sob orientação do Prof. Dr. Luiz Carlos Severo no Laboratório de Micologia e Patologia da Santa Casa Complexo Hospitalar, Porto Alegre, RS.

Endereço para correspondência: Dr. Luiz Carlos Severo. Laboratório de Micologia/Hospital Santa Rita, Santa Casa-Complexo Hospitalar, Annes Dias 285, 90020-090 Porto Alegre, RS.

Fax: 555132148435.

e-mail: severo@ santacasa.tche.br

Recebido em 10/5//2004

Aceito em 06/08/2004
} 


\section{MATERIAL E MÉTODOS}

Foram revisados dezesseis casos de histoplasmoma de 212 prontuários de pacientes com histoplasmose procedentes do Rio Grande do Sul que constam nos arquivos do Laboratório de Micologia, Complexo Hospitalar Santa Casa, Porto Alegre (RS) num período de 25 anos (1977-2002). Os prontuários foram analisados retrospectivamente quanto à idade, sexo, raça, história epidemiológica, sinais e sintomas, condição associada ou predisponente, soromicologia para histoplasmose, tratamento e evolução.

Exame anatomopatológico. Realizados cortes teciduais dos granulomas pulmonares, corados com hematoxilina-eosina (H\&E) - reação tecidual; método de Gomori-Grocott com metenamina argêntica (GMS) - identificação de elementos fúngicos; e técnica de Verhoeff van Gieson (VG) - evidência de cápsula fibrosa.

\section{RESULTAD0S}

Dezesseis (7,5\%) de um total de 212 pacientes apresentavam nódulo pulmonar à radiografia de tórax. Todos os pacientes eram brancos, nove eram do sexo masculino, a idade variou de 10 a 67 anos, média de 50,8 anos. Seis tinham história epidemiológica sugestiva de contato com o fungo. Quatro pacientes tiveram contato esporádico com galinheiro, e dois com fezes de morcegos. Os nódulos variaram de 0,7 a $2,7 \mathrm{~cm}$ de diâmetro. Houve predomínio de nódulo solitário (12; 75\%). Um paciente apresentou dois nódulos; em 3 pacientes foram encontrados três ou mais nódulos. Somente em um paciente a imagem, à radiografia de tórax, sugeria calcificação no interior do nódulo. Os nódulos foram encontrados predominantemente nos lobos inferiores (10; 62,5\%) eno pulmão direito (13; 81,3\%). Treze pacientes eram assintomáticos e os nódulos foram detectados por radiografia (Figura 1) ou tomografia computadorizada ( Figura 2) de tórax. Três pacientes apresentavam sintomas variando de três a seis meses de evolução, respiratórios

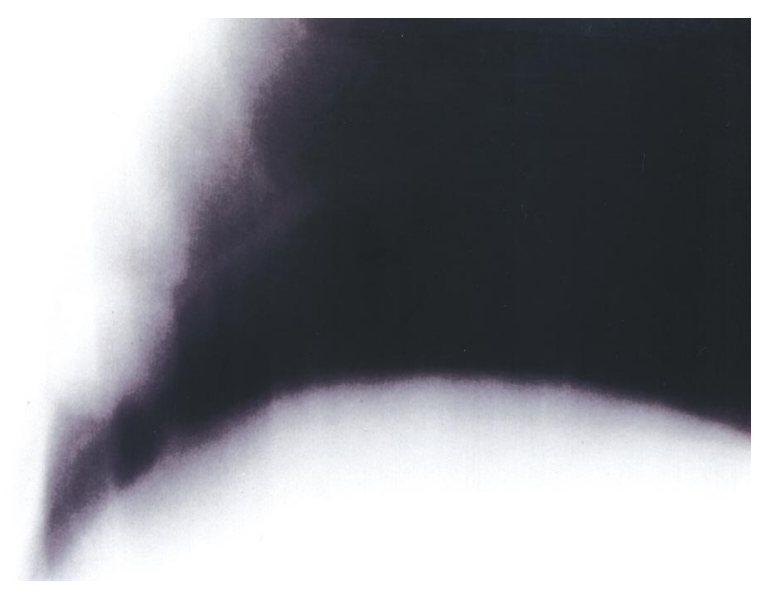

Figura 1 - Caso 1: nódulo $(1,5 \mathrm{~cm})$ na cortical do LID, junto ao seio costofrênico à radiografia de tórax. e/ou sistêmicos, relacionados com doença ativa associada, dois apresentavam carcinoma de pulmão e um, tumor de mediastino. Nenhum apresentava outro foco de infecção fúngica. Mesmo com neoplasia os granulomas não apresentavam evidência histopatológica de reativação. A soromicologia (imunodifusão) foi positiva somente em 2 (20\%) dos 10 pacientes que realizaram 0 exame (Tabela 1). 0 tratamento foi cirúrgico em todos casos. Quatro pacientes receberam itraconazol no pós-operatório.

Padrão histopatológico. Cortes teciduais dos granulomas pulmonares, corados com H\&E, demonstraram granuloma com necrose caseosa em todos os casos. Um nódulo era escavado (Caso 13) e outro apresentava calcificação (Caso 14). A técnica de WG evidenciou a cápsula fibrosa (Figuras 3 e 4). Elementos leveduriformes ovalados pequenos e unibrotantes sugestivos de H. capsulatum foram visualizados na zona central de necrose caseosa com método de GMS (Figura 5) . 0 cultivo foi negativo no único caso em que foi realizado.

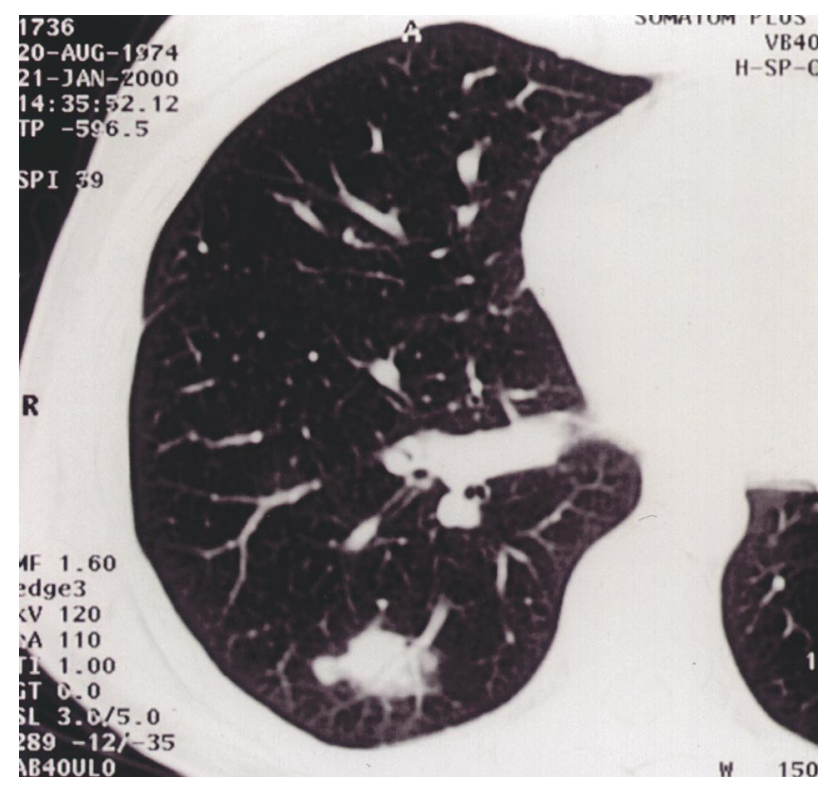

Figura 2 - Caso 12, nódulo $(2,7 \mathrm{~cm})$ subpleural, no segmento inferior do LID à tomografia computadorizada de tórax.

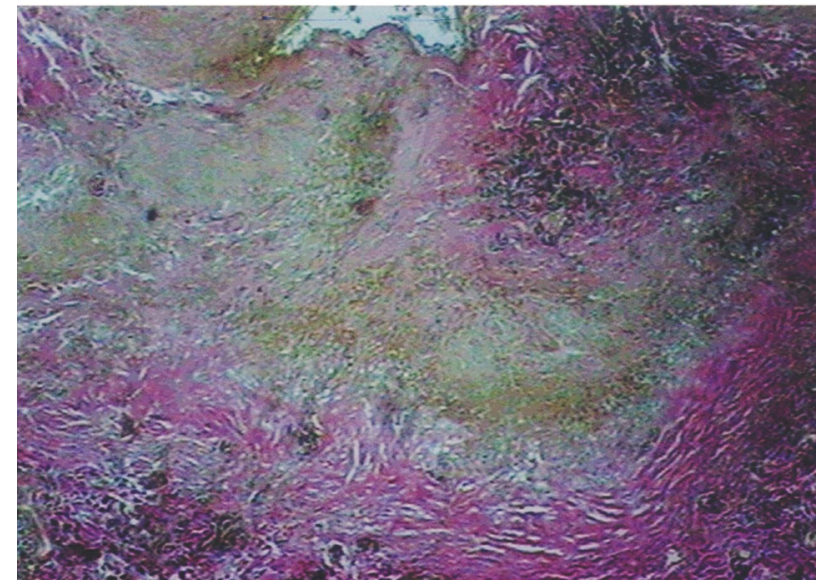

Figura 3 - Nódulo com necrose central e cápsula fibrosa. Coloração de Verhoeff van Gieson (400x). 
Tabela 1 - Características dos histoplasmomas.

\begin{tabular}{lcccccccc}
\hline Caso & Sexo & Idade & Número & Tamanho $(\mathrm{mm})$ & Localização pulmonar & H\&E & GMS & IDh \\
\hline 1 & M & 66 & 1 & 15 & seio costofrênico direito & GNC; CF & + & + \\
2 & F & 62 & 1 & 10 & segmento basal posterior LSD & GNC; CF & + & - \\
3 & M & 45 & 1 & 13 & segmento anterior LSD & GNC; CF & + & - \\
4 & M & 10 & 1 & 20 & segmento infero-posterior direito & GNC; CF & + & - \\
5 & M & 67 & 1 & 9 & pirâmide basal direita & GNC; CF & + & - \\
6 & F & 34 & 3 & $10 ; 10 ; 10$ & LE & GNC; CF & + & + \\
7 & F & 52 & 2 & $15 ; 7$ & segmento basal posterior LSD & GNC; CF & + & - \\
8 & M & 53 & 1 & 20 & segmento lateral LE & GNC; CF & + & - \\
9 & F & 59 & 1 & 12 & segmento superior LD & GNC; CF & + & NR \\
10 & F & 63 & 1 & 13 & segmento posterior LSD & GNC; CF & + & - \\
11 & M & 62 & 1 & 15 & segmento anterior LSD & GNC; CF & + & - \\
12 & F & 25 & 3 & $27 ; 26 ; 20$ & segmento superior LD & GNC; CF & + & - \\
13 & M & 44 & 1 & 13 & segmento basal apical LD & GNC; CF escavado & + & NR \\
14 & F & 52 & 4 & Entre 10 e 18 & LE & GNC; CF & + & NR \\
15 & M & 66 & 1 & 14 & LSD & GNC; CF calcificado & + & NR \\
16 & M & 52 & 1 & 20 & segmento basal posterior LD & GNC; CF & + & NR \\
\hline
\end{tabular}

LSD - lobo superior direito LE - lobo inferior esquerdo LD - lobo inferior direito

GNC - granuloma com necrose caseosa CF - cápsula fibrosa

H\&E - hematoxilina-eosina GMS - Gomori-Grocott com metenamina argêntica

NR - não realizado IDh - imunodifusão para histoplasmose

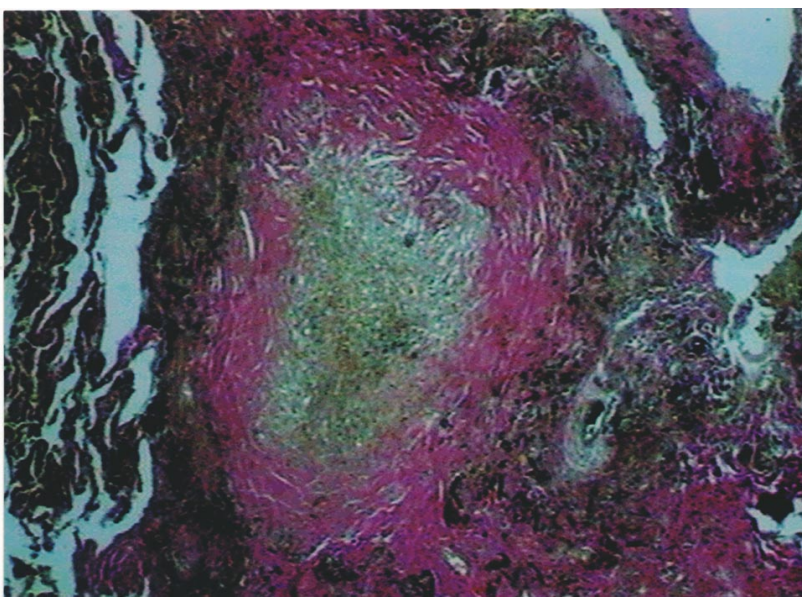

Figura 4 - Nódulo satélite encapsulado. Coloração de Verhoeff van Gieson (400x).

\section{DISCUSSÃ0}

A histoplasmose pode se manifestar de várias formas clínicas dependendo da quantidade de fungo inalado (tempo de exposição e concentração de propágulos fúngicos no ar), imunidade celular do hospedeiro e condições anatômicas do parênquima pulmonar ${ }^{5}$. Histoplasmoma é um achado relativamente incomum relacionado com a cicatrização de lesão primária com formação excessiva de fibrose ${ }^{8}$. Este foco parenquimatoso cicatricial, de dois a quatro milímetros, raramente progride, resultando em um nódulo de crescimento lento que pode alcançar 3 a 4 centímetros em 10 a 20 anos . Alguns achados radiológicos podem auxiliar na identificação deste processo benigno como calcificação central da lesão ou laminações concêntricas de calcificação ${ }^{7}$. A calcificação dos nódulos é um achado mais comum nos histoplasmomas que nos tuberculomas ${ }^{12}$ (Tabela 2). 0 crescimento e a localização

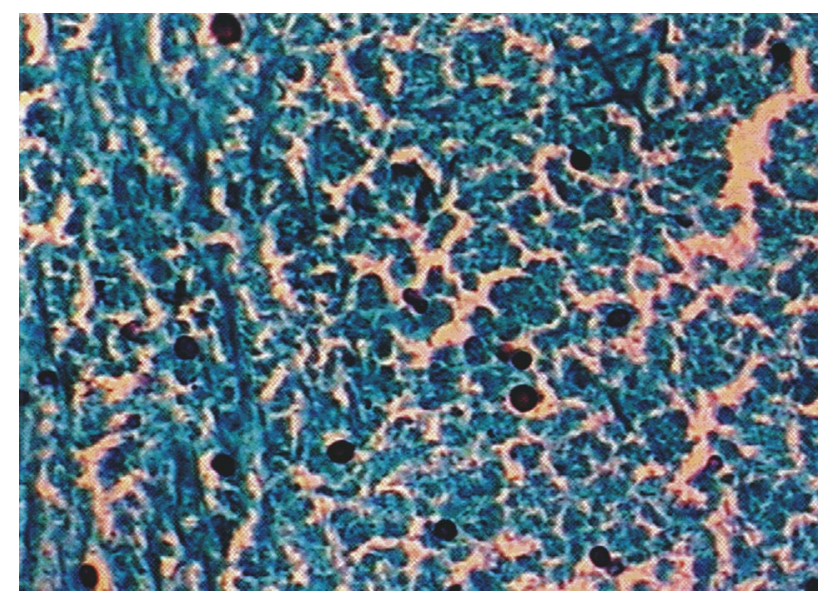

Figura 5 - Presença de elementos leveduriformes pequenos, ovalados, unibrotantes. Coloração de Gomori-Grocott com metenamina argêntica $(400 x)$.

do nódulo assumem importância clínica pelo diagnóstico diferencial com neoplasia e outras doenças granulomatosas não infecciosas ( granulomatose de Wegener, sarcoidose) e infecciosas, como micoses e micobacterioses, especialmente tuberculose ${ }^{618}$.

Tabela 2 - Diagnóstico diferencial entre histoplasmoma e tuberculoma. Achados histológicos predominantes.

\begin{tabular}{lll}
\hline Características & Histoplasmoma & Tuberculoma \\
\hline Número de organismos & muitos & muitos \\
Localização no granuloma & central & central \\
Multiplicidade & solitários & múltiplos \\
Nódulo dominante & sim & não \\
Configuração & redondo & redondo \\
Tipo de necrose & parcialmente caseosa & caseosa \\
Calcificação & presente & presente \\
Tipo de fibrose & esclerosante & fibroblástica \\
Vasculite & ausente & moderada a extensa \\
\hline
\end{tabular}

Fonte: Ulbright TM, Katzenstein ALA ${ }^{18}$ 
Embora infecções fúngicas ou por micobactérias não possam ser diferenciadas sem 0 uso de colorações específicas, há certos padrões histológicos que favorecem um tipo ou outro de infecção (Tabela 2). Os histoplasmomas costumam ser solitários 2 . Quando múltiplos (casos 6, 7, 12,14), apresentam um granuloma dominante e, assim como os paracoccidiodomas múltiplos, simulam granulomatose de Wegener ${ }^{1618}$. 0 diagnóstico é realizado quando da ressecção cirúrgica através de exame histopatológico da peça com a coloração de $H \& E^{101517}$. Os granulomas apresentam mais comumente necrose parcialmente caseosa. São geralmente envoltos por uma cápsula fibrosa esclerosante de 1 a 2 milímetros, apresentam pouca vasculite e freqüentemente contêm cálcio ${ }^{18}$.

A coloração de H\&E não permite a visualização dos elementos fúngicos. É fundamental realizar o método de $\mathrm{GMS}^{19}$. Recomendase dois blocos contendo granulomas necróticos para identificação etiológica. Nos casos em que o GMS não impregna adequadamente nos elementos fúngicos, o tempo de permanência na coloração deve ser prolongad $0^{18}$. Os elementos leveduriformes do $\mathrm{H}$. capsulatum estão presentes nas áreas mais necróticas, no centro do granuloma ${ }^{18}$.

Ausência de crescimento fúngico, mesmo quando este é visível no granuloma, é atribuída ao fato de granulomas antigos não apresentarem organismos viáveis, conferindo ao patologista a responsabilidade de estabelecer a natureza infecciosa ou não do granuloma. Em estudo sobre granulomas necróticos pulmonares, nenhum dos dezenove histoplasmomas apresentou cultivo positivo ${ }^{18}$. Em outra série, de 73 histoplasmomas, cultivo ou testes sorológicos foram negativos em todos os casos onde foram realizados 2 .

No Brasil, há poucos relatos de histoplasmoma pulmonar, a maioria proveniente de estudo de necropsia ${ }^{10}$. Em 42 nódulos pulmonares calcificados, houve evidência histológica de elementos sugestivos de $\mathrm{H}$. capsulatum em quinze (38\%) ${ }^{11}$. Em outro estudo, a causa mais freqüente de nódulos pulmonares, em 36 necropsias, foi infecção por H. capsulatum $(28 ; 77,8 \%)^{1}$. Em 2.411 necrópsias e 40.848 espécimes excisados cirurgicamente de vários sítios, foram encontrados 15 nódulos contendo H. capsulatum no pulmão ${ }^{9}$. Os trabalhos publicados demonstram que nódulos pulmonares têm como causa freqüente infecção por $\mathrm{H}$ capsulatum, especialmente em locais que coincidem com zona endêmica (RS, RJ, MG, SP), apresentando reatividade cutânea elevada à histoplasmina ${ }^{4}$, isolamento do fungo no solo ${ }^{101420}$ e microepidemias relatadas ${ }^{3}$.

Para enfatizar, nódulos solitários de até três centímetros de diâmetro em paciente assintomático devem levantar a hipótese de histoplasmoma, principalmente em regiões sabidamente endêmicas.

\section{REFERÊNCIAS BIBLIOGRÁFICAS}

1. Adad SJ, Machado ME, Batista FC. Histoplasmose residual dos pulmões e linfonodos broncopulmonares em necropsias realizadas em Uberaba, MG. Revista da Sociedade Brasileira de Medicina Tropical 29: 491-496, 1996.

2. Angulo A0, Capecchi Y. Lesiones numulares pulmonares de origen inflamatório. Histoplasmomas. Thorax 1: 27-35, 1973.

3. Cury GC, Diniz Filho A, Costa e Cruz AG, Hobaika ABS. Surto de histoplasmose em Pedro Leopoldo, Minas Gerais, Brasil. Revista da Sociedade Brasileira de Medicina Tropical 34: 483-486, 2001.

4. Fava SC, Fava Netto C. Epidemiologic surveys of histoplasmin and paracoccidioidin sensitivity in Brazil. Revista do Instituto de Medicina Tropical de São Paulo 40: 155-164, 1998.

5. Goodwin RA, Des Prez RM. Pathogenesis and clinical spectrum of histoplasmosis. Southern Medical Journal 66: 13-25, 1973.

6. Goodwin RA, Des Prez RM. Histoplasmosis, state of the art. American Review of Respiratory Disease 117: 929-956, 1978.

7. Goodwin RA, Loyd JE, Des Prez RM. Histoplasmosis in normal hosts. Medicine 60: 231-266, 1981

8. Goodwin RA, Snell JD. The enlarging histoplasmoma. American Review of Respiratory Disease 100: 1-12, 1969.

9. Lemos S. Contribuição ao estudo da histoplasmose humana com especial referência aos nódulos residuais da primo-infecção. Tese de doutorado, Instituto de Medicina Tropical de São Paulo, São Paulo, SP, 1972.

10. Londero AT, Ramos CD. The status of histoplasmosis in Brazil. Mycopathologia 3: 153-156, 1978.

11. Queiroz AC, Siqueira LA. Histoplasmose em material de autópsia. Revista de Patologia Tropical 4: 107-114, 1975.

12. Salzman E. Lung parenchyma infectious granulomas. In: Thomas CC ( ed) Lung calcifications in X-ray diagnosis, Springfield, p.3-77, 1968.

13. Severo LC, Oliveira FM, Irion K, Porto NS, Londero AT. Histoplasmosis in Rio Grande do Sul, Brazil: a 21-year experience. Revista do Instituto de Medicina Tropical de São Paulo 43: 183-187, 2001.

14. Severo LC, Petrillo VF, Camargo JJ, Geyer GR, Porto NS. Acute pulmonary histoplasmosis and first isolation of Histoplasma capsulatum from soil of Rio Grande do Sul, Brasil. Revista do Instituto de Medicina Tropical de São Paulo 28: 51-55, 1986.

15. Severo LC, Picon PD, Londero AT, Rubião HFơ. Histoplasmose aguda. Relato de dois casos. Revista da Associação Médica do Rio Grande do Sul 25: 64-67, 1981.

16. Severo LC, Porto NS, Camargo JJ, Geyer GR. Multiple paracoccidioidomas simulating Wegener's granulomatosis. Mycopathologia 91: 117-119, 1985.

17. Severo LC, Rizzon CFC, Roesch EW, Porto NS. Histoplasmose pulmonar aguda: episódio em casal de adultos. Revista da Associação Médica do Rio Grande do Sul 37: 281-283, 1993.

18. Ulbright TM, Katzenstein ALA. Solitary necrotizing granulomas of the lung The American Journal of Surgical Pathology 4: 13-28, 1980.

19. Wheat LJ. Laboratory diagnosis of histoplasmosis: update 2000. Seminars in Respiratory Infections 16: 131-140, 2001.

20. Zancopé-Oliveira RM, Wanke B. Distribuição das fontes de infecção do Histoplasma capsulatum var. capsulatum em Rio da Prata - Município do Rio de Janeiro (RJ). Revista do Instituto de Medicina Tropical de São Paulo 29: 243-250, 1987. 